\title{
BEAM IMAGING DIAGNOSTICS FOR HEAVY ION BEAM FUSION EXPERIMENTS*
}

\author{
F.M. Bieniosek ${ }^{\#}$, L. Prost, W. Ghiorso \\ LBNL, Berkeley, CA 94720, USA
}

\begin{abstract}
We are developing techniques for imaging beams in heavy-ion beam fusion experiments in the HIF-VNL in 2 to 4 transverse dimensions. The beams in current experiments range in energy from $50 \mathrm{keV}$ to $2 \mathrm{MeV}$, with beam current densities from $<10$ to $200 \mathrm{~mA} / \mathrm{cm}^{2}$, and pulse lengths of 4 to $20 \mu \mathrm{s}$. The beam energy will range up to $10 \mathrm{MeV}$ in near-future beam experiments. The imaging techniques, based on kapton films and optical scintillators, complement and, in some cases, may replace mechanical slit scanners. The kapton film images represent a time-integrated image on the film exposed to the beam. The optical scintillator utilizes glass and ceramic scintillator material imaged by a fast, imageintensified CCD-based camera. We will discuss the techniques, results, and plans for implementation of the diagnostics on the beam experiments.
\end{abstract}

\section{INTRODUCTION}

A number of techniques have been used for diagnosing the transverse spatial and velocity distribution of heavyion beams in the experimental heavy-ion inertial fusion energy program [1]. These diagnostic tools include wire grids, slit scanners, Faraday cups, etc. Recently we have developed the method of imaging beams on kapton film [2]. The beam ions strike the film and degrade its polymer structure. The degradation is visibly evident as a darkening of the film, and acts as a high-resolution indicator of the time-integrated beam current density profile. A drawback of the kapton imaging technique is that the kapton film needs to be replaced for every measurement, and that the image lacks time resolution. As a result we have developed optical diagnostic techniques. These techniques are related to the previously reported gated beam imager (GBI)[3]. The GBI uses a pepper-pot to create beamlets that are imaged directly onto a gated microchannel plate (MCP) and CCD camera. The scintillator-based technique described here replaces the MCP imager with a thin sheet of scintillator material. It is simple, compact, and more flexible, because only the scintillator material is placed in the path of the beam.

Inorganic scintillators are widely used as beam profile monitors, and the mechanism and optical response to an ion beam have been studied extensively [4]. The scintillation yield increases with particle energy; it depends on the ion energy loss per unit path length $\mathrm{dE} / \mathrm{dx}$ and on the ion species. For a given energy the yield is

* Work performed under the auspices of the U.S. Department of Energy by the university of California, Lawrence Berkeley National Laboratory under Contract No. DE-AC03-76F00098..

\#fmbieniosek@lbl.gov somewhat larger for an electron than for a beam ion. This behavior is in contrast to that of kapton film, which strongly discriminates against electrons and low energy ions. But since the beam ion energy is much higher than the maximum energy expected from any stray electrons, the overall yield from beam ions is still expected to be much greater than from the same number of electrons.

\section{OPTICAL BEAM IMAGING}

We have used glass and ceramic (98\% alumina) as scintillator materials. Charge neutralization is provided by a high-transparency ( $80-90 \%$ transmission) metallic mesh placed on or near the surface of the scintillator. Beam ions striking the mesh generate secondary electrons. Since the secondary electron yield is on the order of 10 or greater for the beams of interest, sufficient electrons are created on the mesh to charge-neutralize the insulating surface. In addition, by applying a negative bias to the mesh, stray external electrons are decelerated and deflected away from the scintillator, limiting their contribution to the optical image to negligible levels.

Images on the thin $(0.1 \mathrm{~mm})$ wafer of alumina are viewed from the rear. Typical time response of the optical emission, as measured by a photomultiplier tube (PMT), is shown in Figure 1. The Injector [5] extraction voltage pulse is shown in the upper trace for reference. The two lower traces show the (negative) PMT signal when the exit aperture of HCX is grounded (upper curve) and when the exit aperture of $\mathrm{HCX}$ is at $-30 \mathrm{kV}$ potential. When the exit aperture is at a large negative potential a long tail is observed in the PMT signal, associated with energetic electrons arriving at the scintillator, and as verified by the current draw on the scintillator plate. When the bias is reversed, the tail is no longer present.

The measured rise time of the scintillator is < $40 \mathrm{~ns}$. There is no evidence for saturation or thermal quenching in the scintillator response. Linearity over the range of measured intensities was tested by imaging the same beam in horizontal and vertical slit scans. The local intensities in the two scans differed by an order of magnitude, but the summed intensities of all images in each of the two scans agreed. The lifetime of the scintillator material under intense ion beam bombardment is limited. For an $\mathrm{HCX} \mathrm{K} \mathrm{K}^{+}$beam (kinetic energy $=1.0$ $\mathrm{MeV}, \tau_{\text {pulse }}=4 \mu \mathrm{s}, \mathrm{j}=60 \mathrm{~mA} / \mathrm{cm}^{2}$ ), the optical emission of the material decays with an e-folding rate of $\sim 170$ pulses. As a result, for very intense beams, such as HCX, for long life it is necessary to limit intensity by placing holes or slits upstream of the scintillator. 


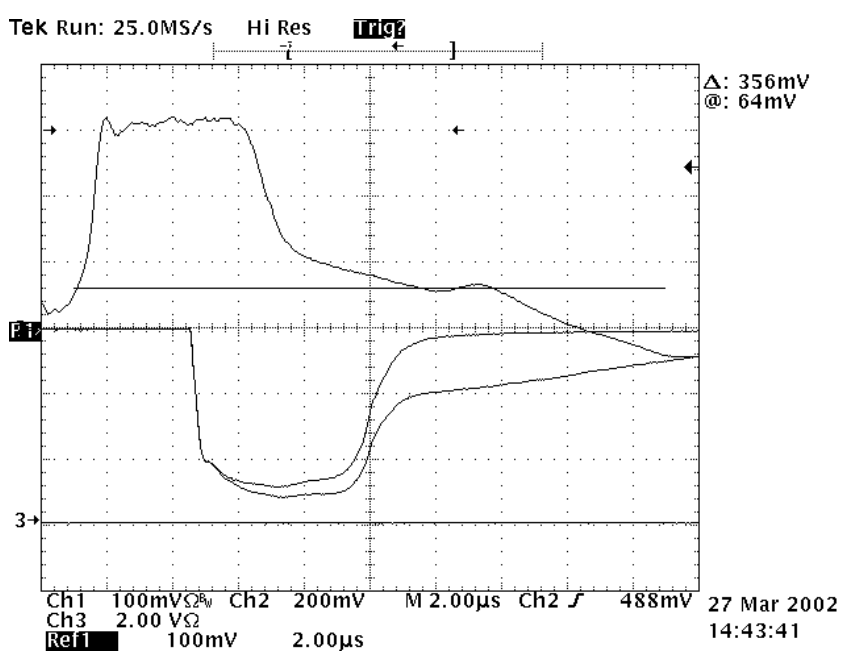

Figure 1. Top: Injector extraction voltage pulse; bottom: optical emission from scintillator at the downstream end of HCX[6] with (upper trace) and without (lower trace) electron suppression.

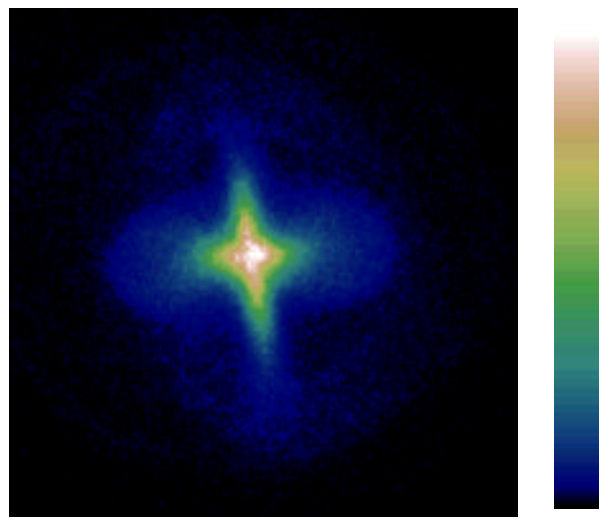

Figure 2. Transverse beam image taken on NTX [7]. The scale is $3 \mathrm{~cm} \mathrm{x} 3 \mathrm{~cm}$. Also shown is the color palette used to represent the images.

\section{BEAM IMAGE DATA}

Time-resolved beam-induced images on the scintillator screen are captured with a Princeton Instruments gated intensified CCD camera viewing the scintillator through a vacuum window. Figure 2 shows a typical beam image taken on NTX. The beam is a final-focus beam with a sharp spot. Images were processed in ImageJ.

In addition to the usual beam distribution in $x-y$ space we have the ability to image the beam in other dimensions of phase space. By interposing horizontal and vertical slits, phase space distributions in $y^{\prime}(x, y), x^{\prime}(x, y)$ are measured; and with the use of a pepper-pot array of pinholes, the distribution in $\mathrm{x}^{\prime}\left(\mathrm{y}^{\prime}(\mathrm{x}, \mathrm{y})\right)$ is measured. Figure 3 shows a series of beam images on the scintillator for a slit at various horizontal locations across the HCX beam. The images are for a 1- $\mu$ s gate in mid-pulse. The dark band in the images is a $0.5-\mathrm{mm}$ support across the slit, located just below the vertical axis of the HCX. The images show the horizontal angular beam spread $\left(x^{\prime}\right)$ as a function of $\mathrm{y}$ for a given slit position $\mathrm{x}$. The structure in

the images represents structure in the transverse particle distribution that is not accessible in a double-slit scan.
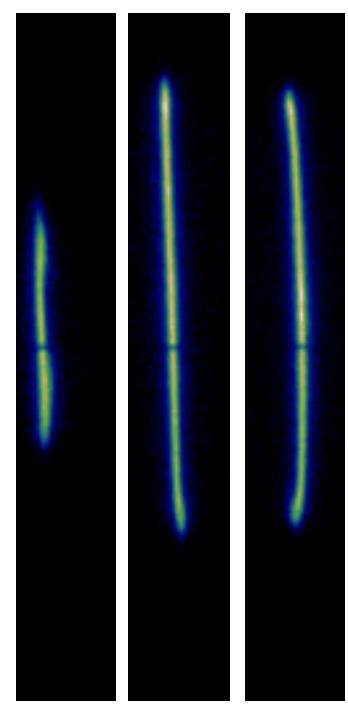

(a)

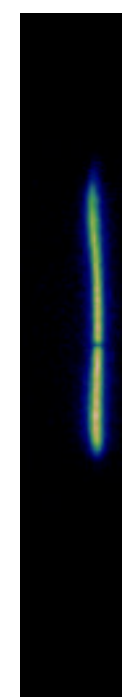

Figure 3. (a) Series of representative images of the beam passing through a slit at various horizontal locations across the beam. (b) Sum of horizontal slit images in a scan.

In a double-slit scan, the signals are summed by a second slit-cup scanned across the first slit such that a line-integrated profile of the beam results. A slit scan can be simulated in the optical data by integrating the optical signal along a vertical or horizontal line. Figure 4 shows a comparison between a line-integrated profile of the beam from the optical data, and the equivalent profile for the same beam measured by moving a slit-cup detector across the beam ("mechanical"). The two curves do not agree exactly because the two diagnostic planes differ $(\Delta \mathrm{z}=4.1 \mathrm{~cm})$. The notch in the optical data corresponds to the location of the support seen in Figure 3.

Table 1: Measured RMS beam parameters

$\begin{array}{lll} & \text { Double Slit } & \text { Optical } \\ \text { a } & 12.3 \mathrm{~mm} & 12.1 \mathrm{~mm} \\ \text { b } & 20.9 \mathrm{~mm} & 20.4 \mathrm{~mm} \\ \text { a' }^{\prime} & -37.9 \mathrm{mrad} & -35.8 \mathrm{mrad} \\ \text { b' }^{\prime} & 43.3 \mathrm{mrad} & 41.6 \mathrm{mrad} \\ \varepsilon_{\mathrm{x}} & 67 \pi \mathrm{mm} \mathrm{mrad} & 76 \pi \mathrm{mm} \mathrm{mrad} \\ \varepsilon_{\mathrm{y}} & 64 \pi \mathrm{mm} \mathrm{mrad} & 71 \pi \mathrm{mm} \mathrm{mrad}\end{array}$

The $2 *$ RMS horizontal and vertical beam size and angle $\mathrm{a}, \mathrm{b}, \mathrm{a}^{\prime}, \mathrm{b}^{\prime}$, and the $4 * \mathrm{RMS}$ emittances $\varepsilon_{\mathrm{x}}$ and $\varepsilon_{\mathrm{y}}$ are calculated in the usual way, and summarized in Table 1 in comparison with mechanical slit scan data for the same beam. The agreement is within experimental errors in the beam size and angle. The emittance measurements are very sensitive to the noise level in the data. Increased noise or scatter in the optical image signal compared to the double-slit scanner is reflected in a higher determination of the emittance. 


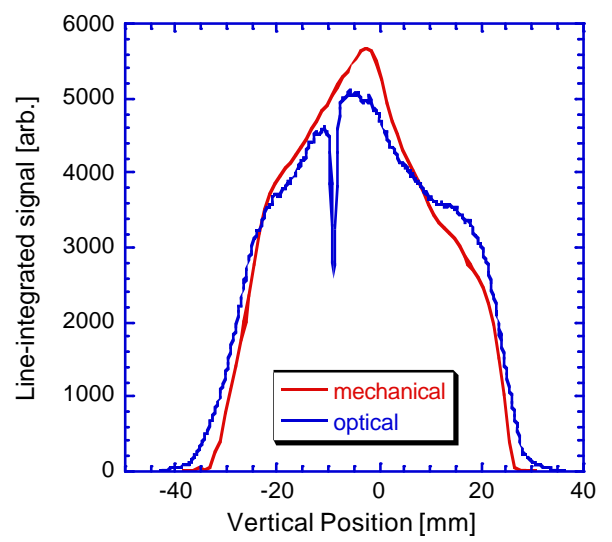

Figure 4. Comparison between a vertical mechanical scan and a simulated optical slit scan for an $80 \%$ fill-factor case on HCX.
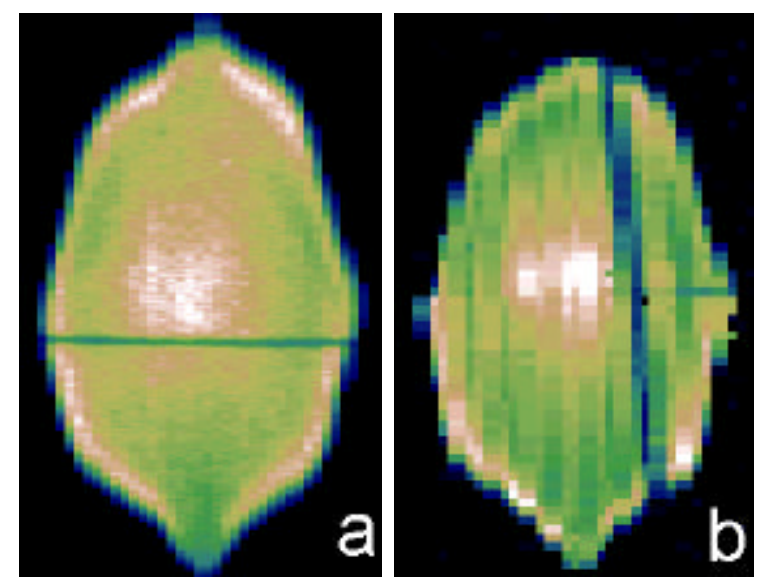

Figure 5. Constructed image of the HCX beam intensity profile based on measured (a) optical slit scan and (b) mechanical crossed-slit measurement. The scale for both images is $3.0 \times 4.68 \mathrm{~cm}$.

Algorithms for reconstruction of the beam profiles in all transverse dimensions have been developed [8]. A typical reconstructed image is shown in Figure 5a. This profile was developed from an optical slit scan (as in Figure 3) using 31 beam puls es. It represents the transverse beam intensity distribution referred to the horizontal slit plane. The assumption of a linear transformation of the beam between the slit and the screen is made. Figure $5 \mathrm{~b}$ shows the equivalent profile of the beam constructed by building up an image point-by-point using a pair of crossed slits, integrated over a $1-\mu \mathrm{s}$ time window in mid pulse. This image required 4096 beam pulses. The sloping dark band in the crossed-slit data is caused by a support across the slit-cup detector entrance slit. Similar images of the moments of the beam distribution, such as the local mean angle, and the local RMS angle spread (which is related to the transverse beam temperature distribution), are also constructed from the optical data.

\section{Optical emission from beam-gas interactions}

Other mechanisms for beam-induced optical emission have been observed. There is a weak optical signal from beam interaction with ambient gas. A stronger emission comes from the gas cloud that is created at a wall when bombarded by the intense ion beam. Images taken through a hole plate show optical signal attributed to this effect through the holes that can be significant for intense or tightly-focused beams. This effect can also be expected to occur at the front surface of the scintillator. The scintillator can be modified to eliminate it by depositing a thin $(150 \mathrm{~nm})$ aluminum layer on the front surface as an optical barrier. In addition, this mechanism provides an alternate imaging technique that is rugged and self-healing. It may prove to be a useful remote diagnostic for beam-wall interactions.

\section{CONCLUSIONS}

Imaging of the beam by optical diagnostic techniques provides a diagnostic tool of great capability and flexibility for HIF-VNL experimental beams. I provides complete, high-resolution two-dimensional images of the beam in as little as a single pulse, in contrast to thousands of beam pulses required by older diagnostic techniques such a crossed-slit scan. It also provides full 4 dimensional beam profile information with the use of slits combined with pinholes.

\section{REFERENCES}

[1] R. O. Bangerter, Nucl. Instrum. Methods Phys. Res. A 464, 17 (2001).

[2] F. M. Bieniosek, et.al., Rev. Sci. Instrum. 73, 2867 (2002).

[3] L. Ahle, H.S. Hopkins, Gated beam imager for heavy ion beams, Proc. 1998 Beam Instrumentation Workshop, SLAC, May 4-7, 1998.

[4] R.B. Murray, and A. Meyer, Phys. Rev. 122, 815 (1961).

[5] F. M. Bieniosek, et. al., 2-MV Injector for HCX, Proc. 2001 Particle Accelerator Conference, Paper WPAH011.

[6] P. A. Seidl, et.al., The High Current Transport Experiment for Heavy Ion Inertial Fusion, Proc. 2003 Particle Accelerator Conference, Paper ROAC001.

[7] S. Yu, et.al., Focusing and neutralization of intense beams, Proc. 2003 Particle Accelerator Conference, Paper TOAA001.

[8] A. Friedman, et.al., Simulation Using Initial 4-D Beam Particle Distributions Synthesized from Experimental Data, Proc. 2003 Particle Accelerator Conference, Paper TOPB006. 